TITLE:

\title{
Tumbling motion of a single chain in shear flow: A crossover from Brownian to non-Brownian behavior
}

$\operatorname{AUTHOR}(S):$

Kobayashi, Hideki; Yamamoto, Ryoichi

\section{CITATION:}

Kobayashi, Hideki ... [et al]. Tumbling motion of a single chain in shear flow: A crossover from Brownian to non-Brownian behavior. Physical Review E 2010, 81(4): 041807.

\section{ISSUE DATE:}

2010-04-26

URL:

http://hdl.handle.net/2433/137184

RIGHT:

(c) 2010 by American Physical Society. 
PHYSICAL REVIEW E 81, 041807 (2010)

\title{
Tumbling motion of a single chain in shear flow: A crossover from Brownian to non-Brownian behavior
}

\author{
Hideki Kobayashi* and Ryoichi Yamamoto ${ }^{\dagger}$ \\ Department of Chemical Engineering, Kyoto University, Kyoto 615-8510, Japan \\ and CREST, Japan Science and Technology Agency, Kawaguchi 332-0012, Japan
}

(Received 13 October 2009; published 26 April 2010)

\begin{abstract}
We present the numerical results for the dynamics of a single chain in steady shear flow. The chain is represented by a bead-spring model and the smoothed profile method is used to accurately account for the effects of thermal fluctuations and hydrodynamic interactions acting on beads due to host fluids. It was observed that the chain undergoes tumbling motions and that its dimensionless frequency $F=6 \pi \eta \sigma^{3} \nu / k_{\mathrm{B}} T$ depends only on the Peclet number Pe with a power law $F \propto \mathrm{Pe}^{\alpha}$, where $k_{\mathrm{B}}$ is the Boltzmann constant, $T$ is the temperature, and $\sigma$ is the diameter of the beads. The exponent $\alpha$ clearly changes from $2 / 3$ to 1 around the critical Peclet number, $\mathrm{Pe}_{\mathrm{c}}$, indicating that the crossover reflects the competition of thermal fluctuation and shear flow. The presented numerical results agree well with our theoretical analysis based on Jeffrey's work.
\end{abstract}

DOI: 10.1103/PhysRevE.81.041807

PACS number(s): 83.80.Rs, 47.57.Ng, 82.20.Wt

\section{INTRODUCTION}

The dynamics of solid particles dispersed in host fluids is an important problem in many different fields of science and engineering. The macroscopic properties of such dispersions (the elastic modulus, viscosity, and thermal and electric conductivities) greatly depend on the dynamics of the particles in the host fluids. In equilibrium states, the dynamics of small dispersed particles are strongly affected by the thermal fluctuations of their host fluids. When flow is imposed, the dynamics are also affected by the flow of the host fluids. Because it is difficult to experimentally analyze these complex particle dynamics, which are coupled both to thermal fluctuations and to fluid flow, numerical simulations are particularly important for understanding the properties of particle dispersions in detail [1].

For a single Brownian chain fluctuating in the shear flow of a Newtonian fluid, it has been suggested that the tumbling frequency $\nu$ is proportional to the shear rate $\dot{\gamma}^{2 / 3}$ [2-9]. This has been experimentally confirmed by Schroeder, Teixeira, Shaqfeh, and Chu [2] for the dynamics of individual DNA molecules in a linear shear flow. Primarily, Smith, Babcock, and $\mathrm{Chu}$ [10] measured the power spectral density (PSD) and the probability distribution function (PDF) of the extension length of each DNA molecule for various Weissenberg numbers $\mathrm{Wi}=\dot{\gamma} \tau$, where $\dot{\gamma}$ is the shear rate and $\tau$ is the relaxation time of the chain orientation. The PSD of polymer extension exhibits no peaks. In subsequent experiments $[2,3]$, however, the focus has been put on the PSD of the orientation angle $\varphi$, where $\varphi=0$ when the DNA molecule lies perfectly in the flow direction. These experimental results support a simple power law, $\nu \tau \propto \mathrm{Wi}^{2 / 3}$, where $\nu$ is the peak frequency of the PSD. The relaxation time $\tau$ is considered to be a constant if the temperature is constant. This leads to $\nu \propto \dot{\gamma}^{2 / 3}$. Similar results have also been obtained in other experiments $[3,4]$, numerical simulations [5,6], and theoretical analyses [7-9].

\footnotetext{
*hidekb@cheme.kyoto-u.ac.jp

†ryoichi@cheme.kyoto-u.ac.jp
}

Although the DNA molecules mentioned above can be considered flexible chains, a quite similar power law was obtained using numerical simulations for a Brownian linear rigid rod as well [2].

For a single non-Brownian (thermally nonfluctuating) flexible chain in shear flow, the tumbling frequency $\nu$ is expected to be proportional to the shear rate $\dot{\gamma}[11]$. A single non-Brownian rigid rod with a finite aspect ratio is known to exhibit a cyclic tumbling motion in shear flow, as described by Jeffrey's equation, $\nu \propto \dot{\gamma}[12-14]$.

From the above experimental findings, one would expect to observe a crossover from Brownian $\left(\nu \propto \dot{\gamma}^{2 / 3}\right)$ to nonBrownian $(\nu \propto \dot{\gamma})$ behavior with increasing shear rate; however, such a clear crossover has not yet been reported. This crossover has not yet been successfully predicted by previous numerical $[5,6]$ and theoretical [7-9] studies where a dispersed chain is treated as an end-to-end vector, namely, as an infinitely thin line. Therefore, the rotational motion of the chain cannot be sustained across $\varphi=0$ without thermal fluctuations. When a thermal fluctuation exists, the orientation of the thin line can fluctuate around $\varphi=0$. This leads to tumbling motions even for a thin line; however, the frequency of the tumbling motion $\nu$ is always proportional to $\dot{\gamma}^{2 / 3}$, regardless of the shear rate [9].

We thus aim to analyze this crossover by use of a direct numerical simulation approach. In the present study, we simulated the tumbling motion of a chain using a smoothed profile method (SPM) that accurately takes into account thermal fluctuations and hydrodynamic interactions [15-18]. The chain is represented by a bead-spring model, wherein each bead is modeled as a spherical object with a finite radius $a$ and undergoes free rotation. Rigid rods or flexible chains are represented with or without a constraint force on bond bending. Apart from the previous numerical models, the presented bead-spring model naturally takes into account the finite thickness of the experimentally used chains or rods. A theoretical analysis has also been developed to understand the mechanisms underlying the crossover. 


\section{METHODS}

\section{A. MODEL}

We solve the dynamics of a single chain in a Newtonian solvent using SPM [15-18]. In this method, boundaries between solid particles and solvents are replaced with a continuous interface by assuming a smoothed profile. This enables us to calculate hydrodynamic interactions both efficiently and accurately, without neglecting many-body interactions. The equation governing a solvent with a density $\rho_{\mathrm{f}}$ and a shear viscosity $\eta$ is a modified Navier-Stokes equation,

$$
\rho_{\mathrm{f}}\left\{\frac{\partial \vec{v}}{\partial t}+(\vec{v} \cdot \vec{\nabla}) \vec{v}\right\}=-\vec{\nabla} p+\eta \vec{\nabla}^{2} \vec{v}+\rho_{\mathrm{f}} \phi \vec{f}_{\mathrm{p}}+\vec{f}_{\text {shear }},
$$

with the incompressible condition $\vec{\nabla} \cdot \vec{v}=0$, where $\vec{v}(\vec{r}, t)$ and $p(\vec{r}, t)$ are the velocity and pressure fields of the solvent, respectively. A smoothed profile function $0 \leq \phi(\vec{r}, t) \leq 1$ distinguishes between the fluid and particle domains, yielding $\phi=1$ in the particle domain and $\phi=0$ in the fluid domain. These domains are separated by thin interstitial regions, the thicknesses of which are characterized by $\xi$. The body force $\phi \vec{f}_{\mathrm{p}}$ is introduced to ensure the rigidity of the particles and the appropriate nonslip boundary condition at the fluid/ particle interface. The mathematical expressions for $\phi$ and $\phi \vec{f}_{\mathrm{p}}$ are detailed in previous papers $[15,16]$. The external force $\vec{f}_{\text {shear }}$ is introduced to maintain a linear shear [19], expressed by

$$
v_{x}= \begin{cases}-\dot{\gamma} y & \left(0<y<L_{y} / 4\right), \\ -\dot{\gamma}\left(-y+\frac{L_{y}}{2}\right) & \left(L_{y} / 4<y<3 L_{y} / 4\right), \\ -\dot{\gamma}\left(y-L_{y}\right) & \left(3 L_{y} / 4<y<L_{y}\right),\end{cases}
$$

where $\dot{\gamma}$ is the shear rate and $L_{y}$ is the system size in the $y$ direction.

In the present study, the chain is represented as either a rigid rod or a flexible chain. We use a bead-spring model consisting of $N$ beads in a single chain. The bead size is sufficient to fit several mesh units. Therefore, it is necessary to consider the torque exerted on the bead. The motion of the $i$ th bead is governed by the following Newton's and Euler's equations of motion with stochastic forces:

$$
\begin{gathered}
M_{i} \frac{d}{d t} \vec{V}_{i}=\vec{F}_{i}^{\mathrm{H}}+\vec{F}_{i}^{\mathrm{P}}+\vec{F}_{i}^{\mathrm{C}}+\vec{G}_{i}^{\mathrm{V}}, \quad \frac{d}{d t} \vec{R}_{i}=\vec{V}_{i}, \\
\vec{I}_{i} \cdot \frac{d}{d t} \vec{\Omega}_{i}=\vec{N}_{i}^{\mathrm{H}}+\vec{G}_{i}^{\Omega},
\end{gathered}
$$

where $\vec{R}_{i}, \vec{V}_{i}$, and $\vec{\Omega}_{i}$ are the position, translational velocity, and rotational velocity of the beads, respectively. $M_{i}$ and $\vec{I}_{i}$ are the mass and moment of inertia, and $\vec{F}_{i}^{\mathrm{H}}$ and $\vec{N}_{i}^{\mathrm{H}}$ are the hydrodynamic force and torque exerted by the solvent on the beads, respectively $[15,16] . \vec{G}_{i}^{\mathrm{V}}$ and $\vec{G}_{i}^{\Omega}$ are the random force and torque, respectively, due to thermal fluctuations. The temperature of the system is defined such that the long-time diffusive motion of dispersed particles reproduces correct behavior $[17,18]$.

$\vec{F}_{i}^{\mathrm{P}}$ represents the potential force due to direct interbead interactions such as Coulombic and Lennard-Jones potentials. We use a bead-spring model as a model of a polymeric chain with a truncated Lennard-Jones potential and a finitely extensible nonlinear elastic (FENE) potential. The truncated Lennard-Jones interaction is expressed in terms of $U_{\mathrm{LJ}}$

$$
U_{\mathrm{LJ}}\left(r_{i j}\right)= \begin{cases}4 \epsilon\left\{\left(\frac{\sigma}{r_{i j}}\right)^{12}-\left(\frac{\sigma}{r_{i j}}\right)^{6}\right\}+\epsilon & \left(r_{i j}<2^{1 / 6} \sigma\right), \\ 0 & \left(r_{i j}>2^{1 / 6} \sigma\right),\end{cases}
$$

where $r_{i j}=\left|\vec{R}_{i}-\vec{R}_{j}\right|$. The parameter $\epsilon$ characterizes the strength of the interactions, and $\sigma$ represents the diameter of the beads. Consecutive beads on a chain are connected by a FENE potential of the form

$$
U_{\mathrm{FENE}}(r)=-\frac{1}{2} k_{\mathrm{c}} R_{0}^{2} \ln \left\{1-\left(\frac{r}{R_{0}}\right)^{2}\right\}
$$

where $r=\left|\vec{R}_{i+1}-\vec{R}_{i}\right|, k_{\mathrm{c}}=30 \epsilon / \sigma^{2}$, and $R_{0}=1.5 \sigma . \vec{F}_{i}^{\mathrm{C}}$ is the constraint force acting on the $i$ th bead due to the bond-angle constraints that cause the chain to form a straight line, and it is used only for the rigid rod case. This is given by

$$
\vec{F}_{i}^{\mathrm{C}}=\frac{\partial}{\partial \vec{R}_{i}}\left(\sum_{\alpha=3}^{N} \vec{\mu}_{\alpha} \cdot \vec{\Psi}_{\alpha}\right)
$$

$$
\vec{\Psi}_{\alpha}=(\alpha-2) \vec{R}_{1}-(\alpha-1) \vec{R}_{2}+\vec{R}_{\alpha}, \quad(\alpha=3, \ldots, N),
$$

where $\vec{\Psi}_{\alpha}=0$ is the constraint condition to be satisfied. $\vec{\mu}_{\alpha}$ is a Lagrange multiplier associated with the intramolecular forces of the constraints chosen such that the constraint condition $\vec{\Psi}_{\alpha}=0$ is satisfied at a time $t+h$, where $\mathrm{h}$ is the time increment of a single simulation step.

\section{B. SIMULATION}

Numerical simulations have been performed in three dimensions with periodic boundary conditions. The lattice spacing $\Delta$ is taken to be the unit of length. The unit of time is given by $\rho_{\mathrm{f}} \Delta^{2} / \eta$, where $\eta=1$ and $\rho_{\mathrm{f}}=1$. The system size is $L_{x} \times L_{y} \times L_{z}=32 \times 16 \times 64$. The other parameters include: $\sigma$ $=4, \xi=2, \epsilon=1, \eta=1, M_{i}=4 \pi a^{3} / 3, N=5$, and $h=0.067$.

In the presented simulations, the Navier-Stokes equation is discretized with a dealiased Fourier spectral scheme in space and with a second-order Runge-Kutta scheme in time. To follow bead motions, the position, velocity and angular velocity of the beads are integrated with the AdamsBashforth scheme.

At $t=0$, the chain aligns along the $x$ axis, which is the shear direction. The run-time of our simulations is about $3520 / \dot{\gamma}$. The range of $k_{\mathrm{B}} T$ is $5.0 \times 10^{-4}<k_{\mathrm{B}} T<1.0$ and that of $\dot{\gamma}$ is $1.0 \times 10^{-3}<\dot{\gamma}<4.0 \times 10^{-2}$.

From the symmetry of the system, to analyze the tumbling motion of a chain, we only have to consider the projected tumbling motion on the $x-y$ plane. We introduce the chain 


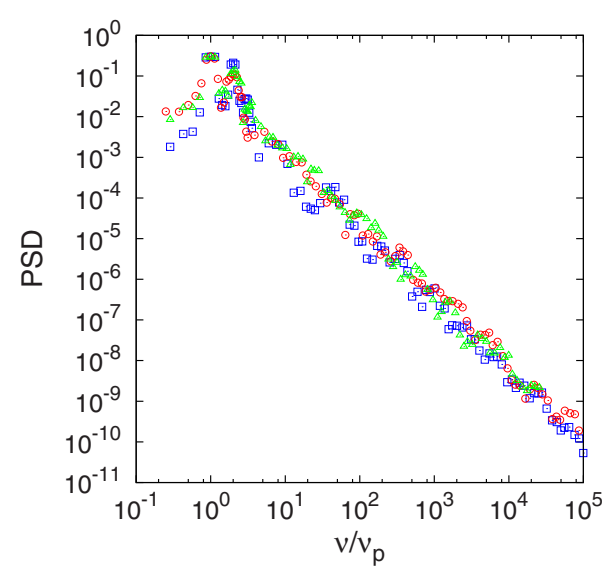

FIG. 1. (Color online) The behavior of PSD as a function of $\nu / \nu_{p}$ for rigid rods at $k_{\mathrm{B}} T=0.0005$ and $\dot{\gamma}=0.001$ (blue $\square$ ), $k_{\mathrm{B}} T=0.006$ and $\dot{\gamma}=0.002$ (red $\bigcirc$ ), and $k_{\mathrm{B}} T=0.01$ and $\dot{\gamma}=0.008$ (green $\triangle$ ).

orientation angle $\varphi$, which is the angle between the $x$ axis and the projected end-to-end vector on the $x-y$ plane.

Evidence of periodic tumbling motion should appear in the PSD per unit time $[2,3]$. Therefore, to investigate the spectral properties of the orientation, we calculate the PSD using a fast Fourier transform. The PSD is expressed as

$$
\operatorname{PSD}(\omega)=\left|\int \varphi(t) \exp (i \omega t) d t\right|
$$

\section{RESULTS}

In Figs. 1 and 2, the PSDs of the chain orientation angle $\varphi$ show a peak at a specific frequency $\nu_{p}$, as shown in previous studies [2,3]. Furthermore, the PSD data obtained at different conditions of shear rate and temperature tend to lie on a single master curve if a normalized frequency $\nu / \nu_{p}$ is used. This is true for both rigid rods and flexible chains. This result implies that the tumbling motion of chains is fully characterized by $\nu_{p}$.

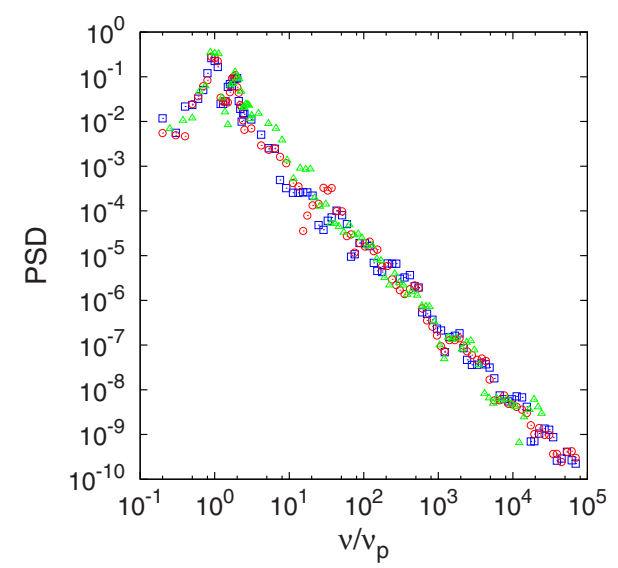

FIG. 2. (Color online) The behavior of PSD as a function of $\nu / \nu_{p}$ for flexible chains at $k_{\mathrm{B}} T=0.0005$ and $\dot{\gamma}=0.001$ (blue $\square$ ), $k_{\mathrm{B}} T=0.006$ and $\dot{\gamma}=0.002$ (red $\bigcirc$ ), and $k_{\mathrm{B}} T=0.01$ and $\dot{\gamma}=0.008$ (green $\triangle$ ).

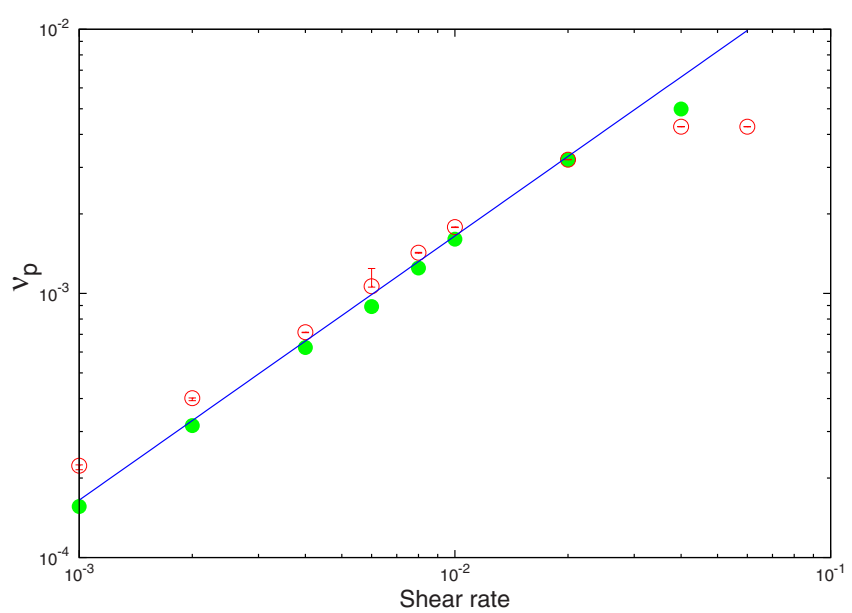

FIG. 3. (Color online) The behavior of $\nu_{p}$ as a function of $\dot{\gamma}$ at $k_{\mathrm{B}} T=0.0005$. Rigid rod (green closed circle) and flexible chain (red open circle). The blue solid line corresponds to the law $\nu_{p} \propto \dot{\gamma}$. Error bars are derived from the half band width of PSD.

In Fig. 3, we find that $\nu_{p}$ follows the law $\nu_{p} \propto \dot{\gamma}$ for $\dot{\gamma}$ $<0.02$ at $k_{\mathrm{B}} T=5.0 \times 10^{-4}$, for both the rigid rod and the flexible chain cases. Meanwhile, Fig. 4 shows $\nu_{p} \propto \dot{\gamma}^{0.68}$ for $\dot{\gamma}$ $<0.02$ at $k_{\mathrm{B}} T=1.0$ for both.

Figure 3 also shows that, for $\dot{\gamma}>0.02, \nu_{p}$ is lower than the frequency expected from the law $\nu_{p} \propto \dot{\gamma}$. P. Bagchi and S. Balachandar have reported that, at a finite Reynolds number approximately equal to 1 , the sphere rotation frequency in a linear shear flow decreases at a much slower rate than $\dot{\gamma} / 2$ [20]. The Reynolds number, Re, is given by $\operatorname{Re}=\rho_{f} \dot{\gamma} \sigma^{2} N / \eta$. In our paper, Re is equal to 1.6 at $\dot{\gamma}=0.02$. We only consider $\nu_{p}$ in the region of $\dot{\gamma} \leq 0.02$, so the effects of finite Reynolds numbers do not influence the results.

We arrange data sets using the Peclet number to consider the effect of competition between shear and fluctuation. The Peclet number is the dimensionless number that relates the rate of shear flow to the rate of thermal fluctuation. In our work, the Peclet number, Pe, and dimensionless frequency, $F$, are expressed as

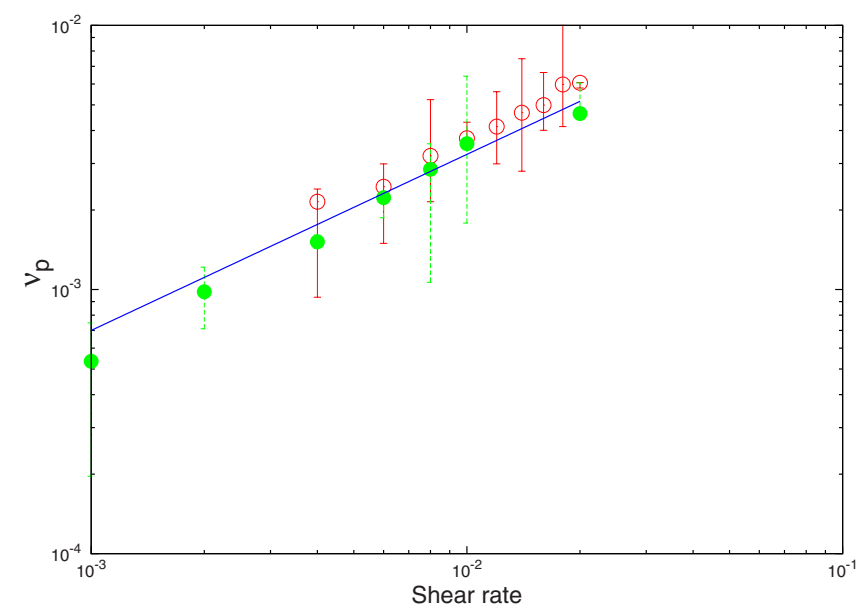

FIG. 4. (Color online) The behavior of $\nu_{p}$ as a function of $\dot{\gamma}$ at $k_{\mathrm{B}} T=1.00$. Rigid rod (green closed circle) and flexible chain (red open circle). The blue solid line corresponds to the law $\nu_{p} \propto \dot{\gamma}^{2 / 3}$. Error bars are derived from the half band width of PSD. 


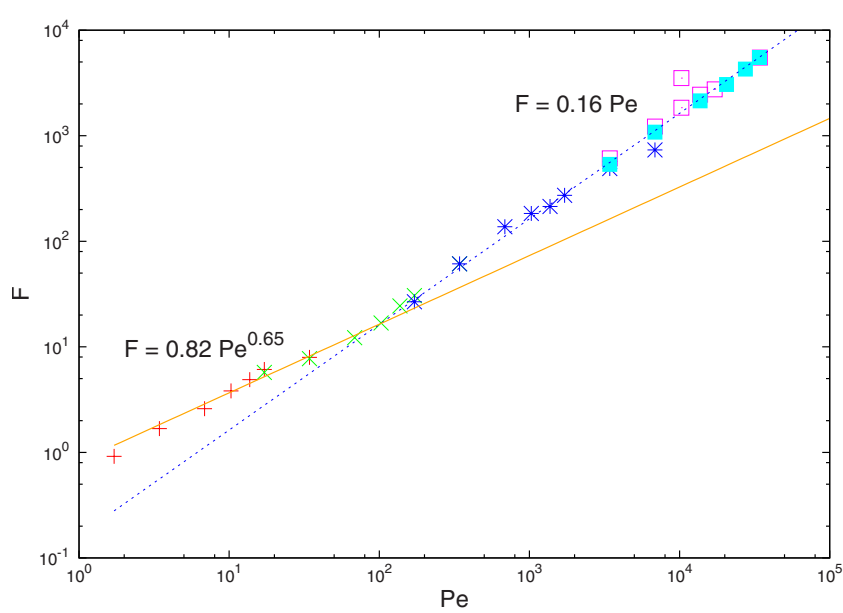

FIG. 5. (Color online) The behavior of $F$ as a function of Pe for a rigid rod. The orange solid and blue dotted lines were calculated from a least-squares fit of the data points. The orange solid line corresponds to $\mathrm{Pe}^{0.65}$. The blue dotted line corresponds to Pe.

$$
\begin{aligned}
& \mathrm{Pe}=\frac{6 \pi \eta \sigma^{3} \dot{\gamma}}{k_{\mathrm{B}} T}, \\
& F=\frac{6 \pi \eta \sigma^{3} \nu_{p}}{k_{\mathrm{B}} T} .
\end{aligned}
$$

We plotted the behavior of $F$ as a function of Pe for the rigid rod and flexible chain cases in Figs. 5 and 6. $F$ was found to depend only on Pe because the data sets have the same value of $F$ with the same value of Pe, even when the shear rates and temperatures are different. In the rigid rod case, $F$ $\propto \mathrm{Pe}^{0.65}$ for $\mathrm{Pe}<106$ and $F \propto \mathrm{Pe}$ for $\mathrm{Pe}>106$. In the flexible chain case, $F \propto \mathrm{Pe}^{0.68}$ for $\mathrm{Pe}<156$ and $F \propto \mathrm{Pe}$ for $\mathrm{Pe}>156$. The behaviors of $F$ for the rigid rod case and the flexible chain case are roughly equal, although the values of the

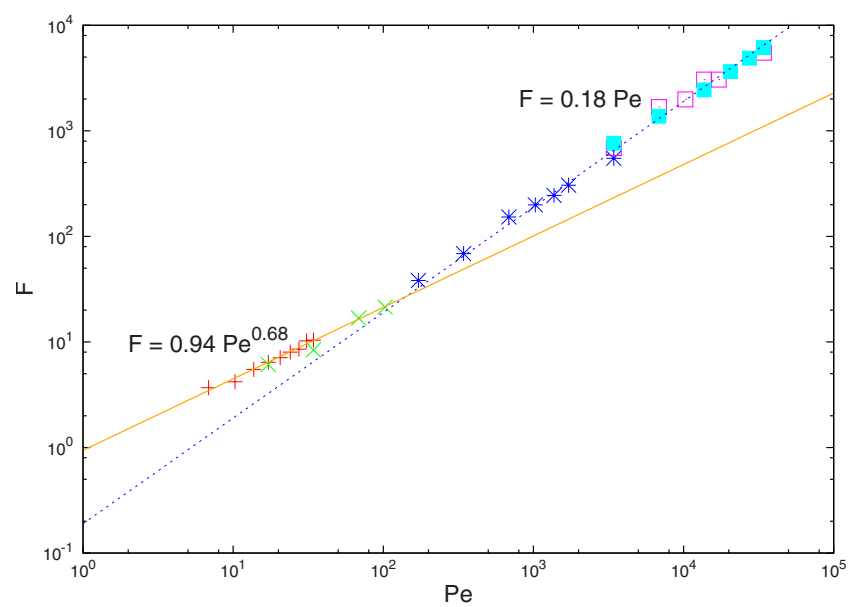

FIG. 6. (Color online) The behavior of $F$ as a function of Pe for a flexible chain. The orange solid and blue dotted lines were calculated from a least-squares fit of the data points. The orange solid line corresponds to $\mathrm{Pe}^{0.68}$. The blue dotted line corresponds to Pe.

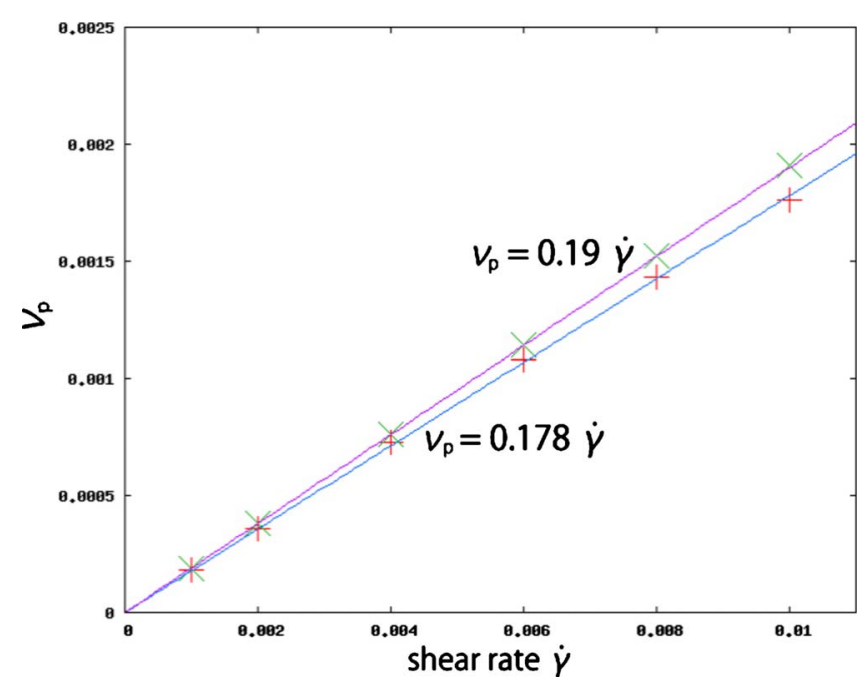

FIG. 7. (Color online) The behavior of $\nu_{p}$ as a function of $\dot{\gamma}$ at $k_{\mathrm{B}} T=0$. Rigid rod $($ red + ), flexible chain $($ green $\times$ ). The data sets for the rigid rods correspond to the law $\nu_{p}=0.178 \dot{\gamma}$. The data sets for the flexible chains correspond to the law $\nu_{p}=0.190 \dot{\gamma}$.

Peclet numbers are different at the boundary where the exponent of Pe changes.

We define the critical value at which the exponent of $\mathrm{Pe}$ drastically changes from almost $2 / 3$ to 1 as the critical Peclet number, $\mathrm{Pe}_{\mathrm{c}} . F \propto \mathrm{Pe}^{2 / 3}$ for $\mathrm{Pe}<\mathrm{Pe}_{\mathrm{c}}$; otherwise, $F \propto \mathrm{Pe}$. When fluctuations dominate the system, the exponent is nearly equal to $2 / 3$. On the other hand, when shear flow dominates, the exponent is exactly equal to 1 .

In order to understand the behavior of $F$ in the limit of $\mathrm{Pe} \rightarrow \infty$, we examine the behavior of $\nu_{p}$ in the limit of $k_{\mathrm{B}} T$ $\rightarrow 0$ (Figure 7). As shown in Figs. 5 and 6, the behavior of $F$ in the region of $\mathrm{Pe}_{\mathrm{c}}<\mathrm{Pe}$ is roughly equal to the behavior of $\nu_{p}$ in the limit of $k_{\mathrm{B}} T \rightarrow 0$. Therefore, we believe that the proportional relation $F \propto \mathrm{Pe}$ can be true across the entire region of $\mathrm{Pe}_{\mathrm{c}}<\mathrm{Pe}$.

\section{DISCUSSION}

\section{A. Comparison with other rfaceesults}

In our work, we calculate the tumbling motion of a single chain for $0.0005<k_{\mathrm{B}} T<1.00$ and $0.001<\dot{\gamma}<0.04$. As reported in Jeffrey's paper [12], which treated non-Brownian particles, $\nu_{p}$ follows the law $\nu_{p} \propto \dot{\gamma}$ at $k_{\mathrm{B}} T=5.0 \times 10^{-4}$, as shown in Fig. 3. In previous papers [5-8] that treated Brownian particles, $\nu_{p}$ follows the law $\nu_{p} \propto \dot{\gamma}^{2 / 3}$ at $k_{\mathrm{B}} T=1.0$, as shown in Fig. 4.

The dimensionless frequency, $F$, depends only on Pe. This Pe dependence on $F$ can be described by a power law, $F$ $\propto \mathrm{Pe}^{\alpha}$. The exponent $\alpha$ drastically changes from $2 / 3$ to 1 at $\mathrm{Pe}_{\mathrm{c}} ; \alpha$ equals $2 / 3$ for $\mathrm{Pe}<\mathrm{Pe}_{\mathrm{c}}$, while $\alpha$ equals 1 for $\mathrm{Pe}_{\mathrm{c}}$ $<\mathrm{Pe}$. In the case of a rigid rod with $N=5, \mathrm{Pe}_{\mathrm{c}} \approx 106$, and $\mathrm{Pe}_{\mathrm{c}} \approx 156$ in the flexible chain case. When fluctuations dominate the system, $F$ follows the law of $F \propto \mathrm{Pe}^{2 / 3}$. On the other hand, when shear flow dominates, $F$ follows the law of $F \propto \mathrm{Pe}$.

Gerashchenko and Steinberg [4] claim that there are two dynamical regimes of polymer motion at $\mathrm{Wi} \gg 1$, depending 
on the polymer extension $R$. When $R \ll R_{\max }$, where $R_{\max }$ is the maximum polymer extension, the tumbling frequency $\nu$ is constant and independent of Wi. On the other hand, when $R \sim R_{\max }, \nu$ is proportional to $\mathrm{Wi}^{2 / 3}$. In Fig. 4, we did not observe $\nu_{p}$ to be independent of $\dot{\gamma}$. We consider the chain length to be too short in our work. The chain cannot keep the coil state along the shear direction for long times. The chain is fully stretched at short notice and is always rotated, although Pe is small.

Previously, Szymczak and Cieplak [21] discussed the conformational dynamics of a single long protein in shear flow and found two characteristic tumbling frequencies, $\nu_{1}$ and $\nu_{2}$. They showed that the higher frequency $\nu_{1}$ follows the law of $\nu_{1} \propto \dot{\gamma}$; however, the lower frequency $\nu_{2}$ follows the law of $\nu_{2} \propto \dot{\gamma}^{2 / 3}$. When the protein is tightly packed, it essentially shows a spherical rotation in shear flow. As a result, $\nu_{1}$ is proportional to $\dot{\gamma}$. The lower frequency, $\nu_{2}$, corresponds to the stretching-collapse cycle; hence, $\nu_{2}$ is proportional to $\dot{\gamma}^{2 / 3}$. Although our results are similar to theirs, the phenomena in our system are essentially different from those in their works because rigid rods cannot fold.

Davoudi and Schumacher [22] analyzed the stretching of polymers in a turbulent flow. It is known that the polymers undergo a coil-stretch transition at $\mathrm{Wi} \approx 1 / 2$ in this system. For $\mathrm{Wi}<1 / 2$, polymers are in the coiled state, and their size distribution is stationary. In contrast, for $\mathrm{Wi}>1 / 2$, the polymers are in the stretched state. Their stretching carries on until their lengths reach the finite extensibility limit or until turbulence stops the growth of the polymers. They found the maximum Lyapunov exponent to be $\lambda \sim \dot{\gamma}^{3 / 2}$. However, Chertkov et al. reported $\lambda \sim \dot{\gamma}^{2 / 3}$ in their work, where $\lambda^{-1}$ is expressed as the mean stretching time scale. Davoudi and Schumacher claimed also that their study could not be compared with the analytic results of Chertkov et al. They defined the shear time scale as $T_{s}=\dot{\gamma}^{-1}$ and the fluctuation time scale as $T_{f}=D^{-1}$, where $D$ is the strength of Gaussian fluctuation. Chertkov analyzed the polymer dynamics in the region of $T_{s} \ll T_{f}$. Davoudi analyzed the polymer dynamics in the region of $\tau_{\eta}<T_{s}$, where $\tau_{\eta}$ is the Kolmogorov time. Notably, Davoudi's work studies a different regime of polymer stretching than the analytic model of Chertkov's work. Chertkov et al. studied in the shear-dominated regime, whereas Davoudi and Schumacher studied in the turbulencedominated regime.

Our work analyzed polymer dynamics in the region of $T_{s} \ll T_{f}$. We do not consider the effect of turbulent flow. We only considered the region with particle Reynolds number $\operatorname{Re}<1.6$, so the effects of finite Reynolds numbers do not influence the results. In Davoudi's work, by contrast, the region of particle Reynolds number in the stretched state is estimated to be $\operatorname{Re}^{\prime}>15$, where $\operatorname{Re}^{\prime}=v L / \nu, \nu$ is kinetic viscosity, $v$ is the root-mean square of the turbulent velocity fluctuation, and $L$ is the mean length of polymer. Therefore, our study cannot be compared with Davoudi's work.

\section{B. Theoretical analysis}

The geometry of the chain in our paper is depicted in Fig. 8. In Jeffrey's work [12], the angle $\varphi$ of non-Brownian rigid

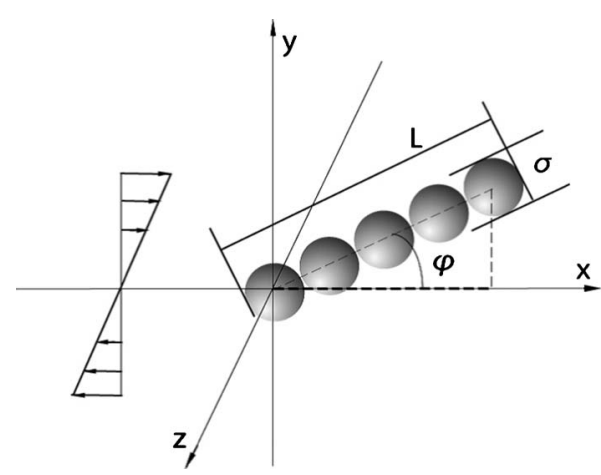

FIG. 8. The geometry of the present simulations.

rods with finite aspect ratios is governed by the equation

$$
\frac{d}{d t} \varphi=-\dot{\gamma} \frac{r^{2}-1}{r^{2}+1} \sin ^{2} \varphi-\dot{\gamma} \frac{1}{r^{2}+1},
$$

where the aspect ratio is $r=L / \sigma$ and $L$ is the length of the chain.

In our work, we consider the equation that governs the angle $\varphi$ of a Brownian rigid rod with a finite aspect ratio. To consider the diffusion of thermal fluctuation, we introduce white noise $\zeta(t)$ into Eq. (12). We can then write down the following equation for a Brownian rigid rod:

$$
\begin{gathered}
\frac{d}{d t} \varphi=-\dot{\gamma} \frac{r^{2}-1}{r^{2}+1} \sin ^{2} \varphi-\dot{\gamma} \frac{1}{r^{2}+1}+2 \sqrt{\frac{D_{r}}{\cos ^{2} \theta}} \zeta, \\
\left\langle\zeta(t) \zeta\left(t^{\prime}\right)\right\rangle=\delta\left(t-t^{\prime}\right),
\end{gathered}
$$

where $D_{r}$ is the rotational diffusion constant. On the basis of the shell model $[23,24]$, the rotational diffusion constant $D_{r}$ for a rigid rod is calculated as

$$
\begin{gathered}
D_{r}=\frac{3[\ln r+d(r)] k_{\mathrm{B}} T}{\pi \eta L^{3}}, \\
d(r)=-0.662+\frac{0.917}{r}-\frac{0.05}{r^{2}} .
\end{gathered}
$$

In the shell model mentioned above, the contour of the macromolecules of arbitrary shape is represented by a shell composed of many identical small beads. The shell model can be adequately modeled by decreasing the size of the beads.

In the case of $D_{r} \ll \dot{\gamma}$, the dynamics of the angle $\varphi$ become decoupled from the angle $\theta$ between the end-to-end vector and the $x-y$ plane because the angle $\theta$ is approximately zero. We can then write down the following equation:

$$
\frac{d}{d t} \varphi=-\dot{\gamma} \frac{r^{2}-1}{r^{2}+1} \sin ^{2} \varphi-\dot{\gamma} \frac{1}{r^{2}+1}+2 \sqrt{D_{r}} \zeta .
$$

For short times, the mean square displacement of $\varphi(t)$ in time $t$ is written as

$$
\left\langle|\varphi(t)-\varphi(0)|^{2}\right\rangle=4 D_{r} t \quad\left(\text { for } D_{r} t \ll 1\right) .
$$

In order to analyze the tumbling motion of a single chain, we only have to consider the dynamics in the stochastic area, 


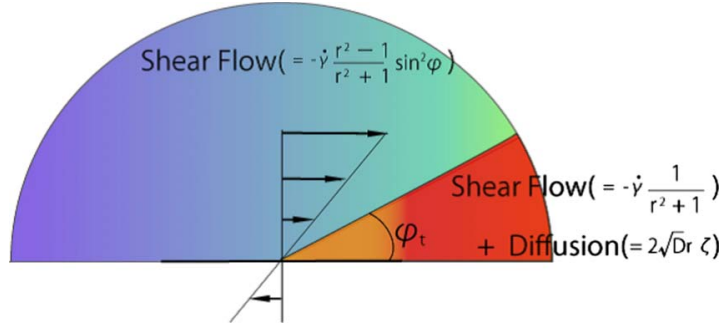

FIG. 9. (Color online) Schematic diagram of two areas and the angle $\varphi_{t}$. The angle $\varphi_{t}$ is the boundary angle of the area dominated by the first term and the area dominated by the second and third terms on the right-hand side of Eq. (17).

where the effect of thermal fluctuation is not negligible. In the case of $D_{r} \ll \dot{\gamma}$, the time required to pass through the stochastic area is sufficiently larger than the time required to pass through the other area. The tumbling motion can only be understood by considering the stochastic area. The stochastic area is dominated by the second and third terms on the right-hand side of Eq. (17). The remaining area is dominated by the first term on the right-hand side of Eq. (17). Figure 9 shows the two areas and the angle $\varphi_{t}$, which is the boundary angle between the two areas. We call the second and third terms on the right-hand side of Eq. (17) the shear and the fluctuation terms, respectively.

\section{Infinite aspect ratio case}

First, we consider Eq. (17) in the limit where $r \rightarrow \infty$. This limit is consistent with treating a chain as an end-to-end vector. We can rewrite Eq. (17) as $\dot{\varphi}=-\dot{\gamma} \sin ^{2} \varphi+2 \sqrt{D_{r}} \zeta$, in which the shear term does not exist. This equation is identical to the equation used in previous works [5-8].

Previous works [4-6] have reported $\varphi_{t} \sim\left(D_{r} / \dot{\gamma}\right)^{1 / 3}$. In the region of $\varphi_{t}<\varphi<\pi$, the shear flow rapidly rotates the orientation of the chain from $\pi$ to $\varphi_{t}$ in a time $t_{l} \approx \dot{\gamma}^{-1}$. In the region of $0<\varphi<\varphi_{t}$, the chain orientation almost aligns along the shear direction. Because the effect of shear becomes sufficiently small in this region, the effect of thermal fluctuations only contributes to rotate the chain orientation from $\varphi_{t}$ to 0 in time $t_{r}=\varphi_{t}^{2} / 4 D_{r} \propto \dot{\gamma}^{-2 / 3}$, as calculated with Eq. (18). In the case of $t_{r}>t_{l}, t_{r}$ dominates the chain tumbling time. We can consider $\nu_{p} \propto t_{r}^{-1}$. Therefore, we conclude from Eqs. (10) and (11) that $F \propto \mathrm{Pe}^{2 / 3}$.

\section{Finite aspect ratio case}

Next, we consider Eq. (17) with a finite aspect ratio, $r$. We can expect $F$ to be proportional to $\mathrm{Pe}^{2 / 3}$ when the fluctuation term dominates in the region $0<\varphi<\varphi_{t}$, as this case agrees with the limit of $r \rightarrow \infty$. Additionally, we can expect $F$ to be proportional to $\mathrm{Pe}$ when the shear term dominates in the region $0<\varphi<\varphi_{t}$ because this case corresponds to the nonBrownian rigid rod. $\mathrm{Pe}_{\mathrm{c}}$ is defined as the Peclet number at which the dominating term in the stochastic area changes from the fluctuation term to the shear term with increasing shear rate.

From Eq. (17), we can write down the corresponding Fokker-Planck equation as

$$
\begin{gathered}
\frac{\partial}{\partial t} P(t, \varphi)+\frac{\partial}{\partial \varphi} J_{\varphi}=0, \\
J_{\varphi}=-\left\{\dot{\gamma} \frac{r^{2}-1}{r^{2}+1} \sin ^{2} \varphi+\dot{\gamma} \frac{1}{r^{2}+1}+2 D_{r} \frac{\partial}{\partial \varphi}\right\} P(t, \varphi),
\end{gathered}
$$

where $P(t, \varphi)$ is the PDF of the angle $\varphi$ and $J_{\varphi}$ is the probability flow. Each term in the braces of Eq. (20) corresponds to a respective term on the right-hand side of Eq. (17).

Next, we focus on the stationary $\operatorname{PDF} P_{\mathrm{st}}(\varphi) \equiv P(t=0, \varphi)$. Because $\partial P_{\mathrm{st}}(\varphi) / \partial t=0$, namely, $\partial J_{\varphi} / \partial \varphi=0$ from Eq. (19), $J_{\varphi}$ is $\varphi$ independent and constant: $J_{\varphi}=-\dot{\gamma}\left[\left(r^{2}-1\right) \sin ^{2} \varphi_{\mathrm{p}} P_{\mathrm{st}}\left(\varphi_{\mathrm{p}}\right)\right.$ $\left.+P_{\mathrm{st}}\left(\varphi_{\mathrm{p}}\right)\right] /\left(r^{2}+1\right)$, where $\varphi_{\mathrm{p}}$ is the angle at which $P_{\mathrm{st}}(\varphi)$ has a peak. We surmise that the angle $\varphi_{t}$ satisfies the equation given by

$$
\frac{J_{\varphi}}{2}=-\dot{\gamma} \frac{\left(r^{2}-1\right) \sin ^{2} \varphi P_{\mathrm{st}}(\varphi)}{r^{2}+1}=-\dot{\gamma} \frac{P_{\mathrm{st}}(\varphi)}{r^{2}+1}-2 D_{r} \frac{\partial P_{\mathrm{st}}(\varphi)}{\partial \varphi} .
$$

By substituting the $J_{\varphi}$ expressed by $\varphi_{\mathrm{p}}$ into Eq. (21), the relation of $\varphi_{t}$ to $\varphi_{\mathrm{p}}$ is given as

$$
\begin{gathered}
-\dot{\gamma} \frac{\left(r^{2}-1\right) \sin ^{2} \varphi_{\mathrm{p}} P_{\mathrm{st}}\left(\varphi_{\mathrm{p}}\right)+P_{\mathrm{st}}\left(\varphi_{\mathrm{p}}\right)}{r^{2}+1} \\
=-2 \dot{\gamma} \frac{\left(r^{2}-1\right) \sin ^{2} \varphi_{t} P_{\mathrm{st}}\left(\varphi_{t}\right)}{r^{2}+1} .
\end{gathered}
$$

The angle $\varphi_{t}$ is calculated by solving Eq. (22). $P_{\mathrm{st}}\left(\varphi_{t}\right)$ is expanded in powers $\Delta \varphi=\varphi_{t}-\varphi_{\mathrm{p}}$. When we neglect the second order of $\Delta \varphi$ and higher, we can relate $\varphi_{t}$ to $\varphi_{\mathrm{p}}$ as

$$
\varphi_{t}=\sqrt{\frac{1}{2}\left(\varphi_{\mathrm{p}}^{2}+\frac{1}{r^{2}-1}\right)} .
$$

It should be noted that $\varphi_{t}, \varphi_{\mathrm{p}} \ll 1$. We can estimate $\varphi_{t}$ with the angle $\varphi_{\mathrm{p}}$.

Next, we attempt to calculate the analytical form of $\varphi_{\mathrm{p}}$. In the case of $D_{r} / \dot{\gamma} \ll 1$, the formal solution for $P_{\text {st }}(\varphi)$ is given by

$$
P_{\mathrm{st}}(\varphi)=C_{1} \int_{0}^{\pi} d \psi \exp \left(-\frac{\dot{\gamma}}{4 D_{r}} f(\psi, \varphi)\right)
$$

$$
f(\psi, \varphi)=\psi-\left(1-\frac{2}{r^{2}+1}\right) \sin \psi \cos (\psi-2 \varphi),
$$

where $C_{1}$ is determined from the normalization condition, $\int_{0}^{\pi} P_{\mathrm{st}}(\varphi) d \varphi=1$. In the limit of $r \rightarrow \infty$, it is known that $\varphi_{\mathrm{p}}$ $=\left(D_{\mathrm{r}} / \dot{\gamma}\right)^{1 / 3}$ [6]. When $r$ is finite, $\varphi_{\mathrm{p}}$ is different from $\left(D_{\mathrm{r}} / \dot{\gamma}\right)^{1 / 3}$ because the shear term influences $P_{\mathrm{st}}(\varphi)$.

To estimate the effect of the shear term, we introduce the times $t_{2}^{\prime}$ and $t_{3}^{\prime}$, where $t_{2}^{\prime}$ and $t_{3}^{\prime}$ represent the times required to pass through the region $0<\varphi<\left(D_{\mathrm{r}} / \dot{\gamma}\right)^{1 / 3}$, depending only on the shear term and the fluctuation term, respectively. $t_{2}^{\prime}$ is estimated by dividing $\left(D_{\mathrm{r}} / \dot{\gamma}\right)^{1 / 3}$ by the shear term as 


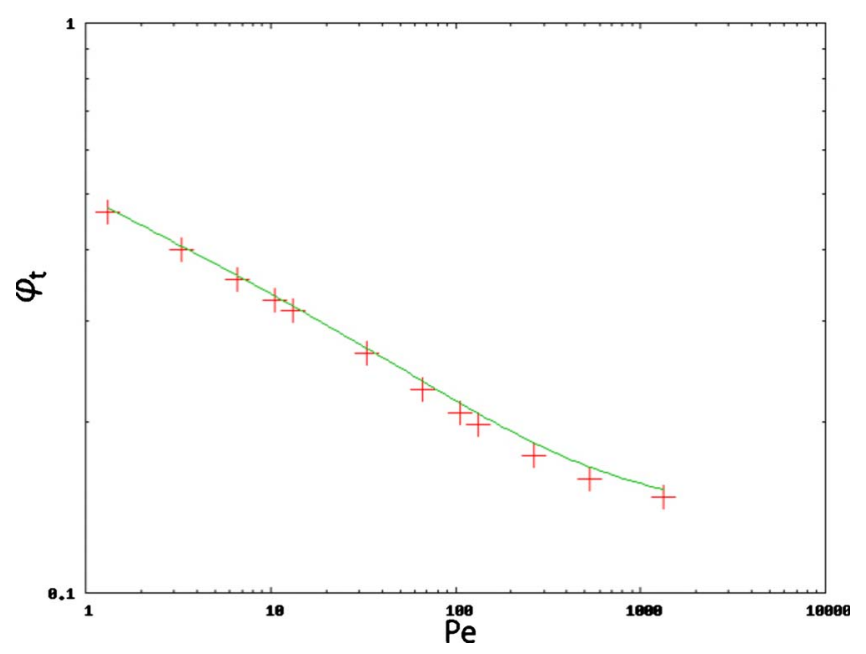

FIG. 10. (Color online) The behavior of $\varphi_{t}$ as a function of Pe. $\varphi_{t^{\prime}}$ is $\varphi_{t}$ calculated with $\varphi_{\mathrm{p}}$, obtained from numerical integration (red + ). The green solid line corresponds to the behavior of $\varphi_{t^{\prime \prime}}$, where $\varphi_{t^{\prime \prime}}$ is $\varphi_{t}$, obtained from $\varphi_{\mathrm{p}}=\left[t_{2}^{\prime} /\left(t_{2}^{\prime}+t_{3}^{\prime}\right)\right] \times\left(D_{\mathrm{r}} / \dot{\gamma}\right)^{1 / 3}$.

$$
t_{2}^{\prime}=\frac{\left(r^{2}+1\right) D_{\mathrm{r}}^{1 / 3}}{\dot{\gamma}^{4 / 3}}
$$

and $t_{3}^{\prime}$ is estimated by Eq. (18) as

$$
t_{3}^{\prime}=\frac{1}{4 D_{\mathrm{r}}^{1 / 3} \dot{\gamma}^{2 / 3}} .
$$

Because the Brownian chain is rotated by both the shear term and the fluctuation terms, we expect that $\varphi_{\mathrm{p}}$ is given by

$$
\begin{gathered}
\varphi_{\mathrm{p}}=C^{\prime}(r, \mathrm{Pe})\left(\frac{D_{\mathrm{r}}}{\dot{\gamma}}\right)^{1 / 3}, \\
C^{\prime}(r, \mathrm{Pe}) \equiv \frac{t_{2}^{\prime}}{t_{2}^{\prime}+t_{3}^{\prime}}=\left\{1+\frac{1}{4 r^{2}+1}\left\{\frac{r^{3}}{18[\ln r+d(r)]} \mathrm{Pe}\right\}^{2 / 3}\right\}^{-1} .
\end{gathered}
$$

For large $\dot{\gamma} / D_{r}$ where the present theoretical analysis is valid, $C^{\prime}(r, \mathrm{Pe}) \sim t_{2}^{\prime} / t_{3}^{\prime}$ finally approaches zero. We confirmed, however, that $t_{2}^{\prime} / t_{3}^{\prime}$ still remains finite $(\sim 1)$ around $\mathrm{Pe}_{\mathrm{c}} \approx 100$, where $\dot{\gamma} / D_{r} \approx 1000$.

Figure 10 shows the behaviors of $\varphi_{t^{\prime}}$ and $\varphi_{t^{\prime \prime}}$ as a function of Pe, where $\varphi_{t^{\prime}}$ is $\varphi_{t}$ calculated with Eq. (23) and $\varphi_{\mathrm{p}}$, which is obtained from the numerical integration of Eq. (24), and $\varphi_{t^{\prime \prime}}$ is $\varphi_{t}$ calculated with Eq. (23) and $\varphi_{\mathrm{p}}$, which is expressed as Eq. (28). In this figure, it is shown that Eq. (28) is established because the behavior of $\varphi_{t^{\prime}}$ agrees well with $\varphi_{t^{\prime \prime}}$ for Pe $\leq 100$. We can obtain $\varphi_{t}$ by substituting Eq. (28) into Eq. (23). Therefore, $\varphi_{t}$ is given by

$$
\varphi_{t}=C(r, \mathrm{Pe})\left(\frac{D_{\mathrm{r}}}{\dot{\gamma}}\right)^{1 / 3},
$$

$$
C(r, \mathrm{Pe})=\sqrt{\frac{1}{2}\left\{C^{\prime 2}+4\left(\frac{r^{2}+1}{r^{2}-1}\right) \frac{1-C^{\prime}}{C^{\prime}}\right\}} .
$$

We consider that $F$ is proportional to $\mathrm{Pe}^{2 / 3}$ when the effect of thermal fluctuation is more significant than the effect of shear flow in the region of $0<\varphi<\varphi_{t}$ and that $F$ is proportional to $\mathrm{Pe}$ in the opposite case. $t_{2}$ is the time required to pass through the region $0<\varphi<\varphi_{t}$ by the shear term,

$$
t_{2}=\frac{\left(r^{2}+1\right) C D_{\mathrm{r}}^{1 / 3}}{\dot{\gamma}^{4 / 3}} .
$$

$t_{3}$ is the time required to pass through the region $0<\varphi<\varphi_{t}$ by the fluctuation term,

$$
t_{3}=\frac{C^{2}}{4 D_{\mathrm{r}}^{1 / 3} \dot{\gamma}^{2 / 3}} .
$$

It is thought that $\mathrm{Pe}_{\mathrm{c}}$ is the Peclet number that satisfies $t_{2}$ $=t_{3}$. If we obtain the value of $r$, we know the value of $\mathrm{Pe}_{\mathrm{c}}$ because both $t_{2}$ and $t_{3}$ are functions of Pe and $r$. Our results show that $\mathrm{Pe}_{\mathrm{c}} \approx 115$ at $r=5$. In the numerical results obtained from our work, $\mathrm{Pe}_{\mathrm{c}}=106$. The analytical result agrees well with our numerical result. Moreover, the numerical condition $D_{\mathrm{r}} \ll \dot{\gamma}$ is satisfied because $D_{\mathrm{r}} / \dot{\gamma} \approx 10^{-3} \ll 1$ at $\mathrm{Pe} \approx 100$. Therefore, the considerations in this section are reasonable in the region near $\mathrm{Pe}_{\mathrm{c}}$.

The considerations in this section agree well with those of previous experimental results [2-4,10,14]. In experimental works that measured the frequencies of DNA rotation $[2-4,10]$, the DNA molecules contained roughly 400 persistence lengths. The persistence length is thought to correspond to $r$. Thus, we conclude that $\mathrm{Pe}_{\mathrm{c}} \approx 516$. These experiments were carried out in the region of $\mathrm{Pe} \ll \mathrm{Pe}_{\mathrm{c}}$, and $F$ is proportional to $\mathrm{Pe}^{2 / 3}$. In experimental work that measured the frequencies of freely rotating rigid dumbbells [14], the aspect ratio $r$ of the rigid dumbbell corresponds to 2 and $\mathrm{Pe}_{\mathrm{c}} \approx 60$. The experiments were carried out in the region of $\mathrm{Pe}_{\mathrm{c}} \ll \mathrm{Pe}$, and $F$ was proportional to Pe. From these results, the considerations in this section are reasonable.

\section{CONCLUSION}

In our work, we calculated the tumbling motion of a single chain using an SPM that takes into account thermal fluctuations and hydrodynamic interactions for 0.0005 $<k_{\mathrm{B}} T<1.00$ and $0.001<\dot{\gamma}<0.02$. We conclude that the dimensionless frequency, $F$, depends only on Pe. The dependence of $F$ can be described by a power law $F \propto \mathrm{Pe}^{\alpha}$. The exponent $\alpha$ sharply changes from $2 / 3$ to 1 on $\mathrm{Pe}_{\mathrm{c}}$. In the case of a rigid rod with $N=5, \mathrm{Pe}_{\mathrm{c}} \approx 106$, and in the case of a flexible chain with $N=5, \mathrm{Pe}_{\mathrm{c}} \approx 156$. The behavior of $F$ for both cases is similar, while only the values of $\mathrm{Pe}_{\mathrm{c}}$ are different from each other.

We have presented $F$ to be proportional to $\mathrm{Pe}^{2 / 3}$ when the third term on the right-hand side of Eq. (17) dominates in the region $0<\varphi<\varphi_{t}$, and $F$ is proportional to $\mathrm{Pe}$ when the 
second term of Eq. (17) dominates in the region $0<\varphi<\varphi_{t}$. We have estimated the angle $\varphi_{t}$ at which the fist term of $J_{\varphi}$, expressed as Eq. (20), is comparable to the sum of the second and third terms of $J_{\varphi}$, expressed as Eq. (20).

A proposed mechanism for this exponent change is that the effect of thermal fluctuation is more significant than the effect of shear flow only for $0<\varphi<\varphi_{t}$, whereas in the other case, the effect of thermal fluctuation is negligible. The former contribution leads to $F \propto \mathrm{Pe}^{2 / 3}$, and the latter contribution leads to $F \propto \mathrm{Pe}$.

\section{ACKNOWLEDGMENT}

The authors would like to express their gratitude to Dr. T. Murashima, Dr. Y. Nakayama, Dr. K. Kim, and Dr. T. Iwashita for useful comments and discussions.
[1] K. Kamata, T. Araki, and H. Tanaka, Phys. Rev. Lett. 102, 108303 (2009).

[2] C. M. Schroeder, R. E. Teixeira, E. S. G. Shaqfeh, and S. Chu, Phys. Rev. Lett. 95, 018301 (2005).

[3] R. E. Teixeira, H. P. Babcock, E. S. G. Shaqfeh, and S. Chu, Macromolecules 38, 581 (2005).

[4] S. Gerashchenko and V. Steinberg, Phys. Rev. Lett. 96, 038304 (2006).

[5] A. P. A. Celani and K. Turitsyn, Europhys. Lett. 70, 464 (2005).

[6] A. Puliafito and K. Turitsyn, Physica D 211, 9 (2005).

[7] M. Chertkov, I. Kolokolov, V. Lebedev, and K. Turitsyn, J. Fluid Mech. 531, 251 (2005).

[8] K. S. Turitsyn, J. Exp. Theor. Phys. 105, 655 (2007).

[9] R. G. Winkler, Phys. Rev. Lett. 97, 128301 (2006).

[10] D. E. Smith, H. P. Babcock, and S. Chu, Science 283, 1724 (1999).

[11] S. Yamamoto and T. Matsuoka, J. Chem. Phys. 98, 644 (1993).

[12] G. B. Jeffery, Proc. R. Soc. London, Ser. A 102, 161 (1922).
[13] F. P. Bretherton, J. Fluid Mech. 14, 284 (1962).

[14] K. Takamura, J. Colloid Interface Sci. 83, 516 (1981).

[15] Y. Nakayama and R. Yamamoto, Phys. Rev. E 71, 036707 (2005).

[16] Y. Nakayama, K. Kim, and R. Yamamoto, Eur. Phys. J. E 26, 361 (2008).

[17] T. Iwashita, Y. Nakayama, and R. Yamamoto, J. Phys. Soc. Jpn. 77, 074007 (2008).

[18] T. Iwashita and R. Yamamoto, Phys. Rev. E 79, 031401 (2009).

[19] J. Schumacher and B. Eckhardt, Europhys. Lett. 52, 627 (2000).

[20] P. Bagchi and S. Balachandar, Phys. Fluids 14, 2719 (2002).

[21] P. Szymczak and M. Cieplak, J. Chem. Phys. 127, 155106 (2007).

[22] J. Davoudi and J. Shumacher, Phys. Fluids 18, 025103 (2006).

[23] M. M. Tirado and J. G. de la Torre, J. Chem. Phys. 73, 1986 (1980).

[24] M. M. Tirado, J. Chem. Phys. 81, 2047 (1984). 